\title{
HIGH INTENSITY ION BEAM INJECTION INTO THE 88-INCH CYCLOTRON
}

\author{
D. Wutte ${ }^{1}$, D.J. Clark ${ }^{1}$, B. Laune ${ }^{2}$, M.A. Leitner ${ }^{1}$, C.M. Lyneis ${ }^{1}$, \\ ${ }^{1}$ Lawrence Berkeley National Laboratory, Berkeley, USA, \\ ${ }^{2}$ Institut de Physique Nucleaire 91406 Orsay Cedex France
}

\begin{abstract}
Low cross section experiments to produce super-heavy elements have increased the demand for high intensity heavy ion beams at energies of about $5 \mathrm{MeV} /$ nucleon at the 88-Inch Cyclotron at the Lawrence Berkeley National Laboratory. Therefore, efforts are underway to increase the overall ion beam transmission through the axial injection line and the cyclotron. The ion beam emittance has been measured for various ion masses and charge states. Beam transport simulations including space charge effects were performed for both the injection line and the ion source extraction. The relatively low nominal injection voltage of $10 \mathrm{kV}$ was found to be the main factor for ion beam losses, because of beam blow up due to space charge forces at higher intensities. Consequently, experiments and simulations have been performed at higher injection energies, and it was demonstrated that the ion beams could still be centered in the cyclotron at these energies. Therefore, the new injector ion source VENUS and its ion beam transport system (currently under construction at the 88-Inch Cyclotron) are designed for extraction voltages up to $30 \mathrm{kV}$.
\end{abstract}

\section{INTRODUCTION}

In FY 2000 34\% (Oct. 99 to Sept. 00) of the total cyclotron beam time was allocated to high intensity ion beam experiments at energies near the Coulomb barrier (about 5MeV/u) for the Berkeley Gas Spectrometer [1] and other nuclear chemistry experiments. Besides several high intensity ${ }^{86} \mathrm{Kr}$ (350 pnA) runs totaling $870 \mathrm{~h}$ of accelerator time, ${ }^{24,25,26} \mathrm{Mg}$ (up to $700 \mathrm{pnA}$ ), ${ }^{50} \mathrm{Ti}$ (300 $\mathrm{pnA}$ ), and ${ }^{64} \mathrm{Ni}$ (200 pnA) have been accelerated for above mentioned experiments, each run typically lasting between 52 and 150 hours. These high intensity runs have unveiled a variety of issues related to the production and acceleration of high intensity ion beams.

Figure 1 summarizes the current performance of the 88-Inch Cyclotron in respect to the maximum extracted ion beam intensities for various beams from $\mathrm{H}$ to Xe. For the lowest cross section experiments with BGS (below $10 \mathrm{pb}$ ), ion beam intensities of $1.6 \mathrm{p} \mu \mathrm{A}$ throughout the whole mass range (intensity level indicated in the figure with a solid line) would be desired.

The maximum extracted beam intensity out of the 88Inch Cyclotron decreases with increasing ion mass. For light and medium mass ions up to $\mathrm{Ar}$ the $1.6 \mathrm{p} \mu \mathrm{A}$ requirement can be met, but for heavier masses the intensities are lower than desired $\left(400 \mathrm{pnA} \mathrm{Kr}^{19+}\right.$, $60 \mathrm{pnA} \mathrm{Xe}{ }^{28+}$ ).

There are many factors that contribute to this behavior: First, higher charge states are used for the acceleration of heavier ions in order to reach $5 \mathrm{MeV} / \mathrm{u}$ in the cyclotron. For light ion masses the ECR ion sources can produce more current than needed, and it is possible to compensate for any losses in the beam lines and the cyclotron. At the medium ion masses space charge effects in the cyclotron injection beam line increasingly limit the maximum extracted current. For the heaviest ions, which require a very high charge state, the current is limited by the ion source performance.

Secondly, the oven efficiencies and the high cost of the rare isotopes often affect the metal ion beam intensities. The third factor is attributed to losses due to beam interaction with residual gas, which increase rapidly for high charge state heavy ion beams [2].

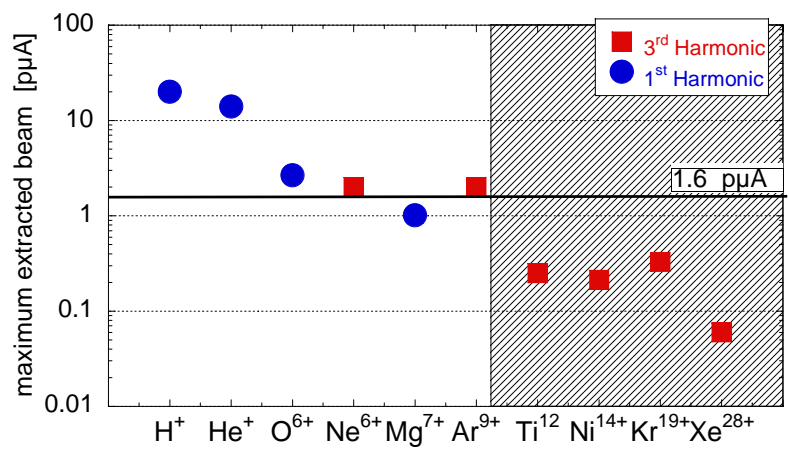

Figure 1: Maximum extracted ion beam current out of the 88-Inch Cyclotron for various ion beams.

\section{HIGH INTENSITY ION BEAM PRODUCTION}

\subsection{Injector Ion Sources}

The 88-Inch Cyclotron is fed by two ECR ion sources. The LBL ECR (6.4 GHz) was built in 1984 and is mainly used for light and medium charge state heavy ion beams [3]. The AECR-U (14 and $10 \mathrm{GHz}$ ) is used for the more challenging beams: medium (e.g. $\mathrm{Kr}^{19+}$ ) and low intensity high (e.g. $\mathrm{Xe}^{38+}, \mathrm{Bi}^{41+}$ ) charge state heavy ion beams [4]. A new injector ion source, the superconducting ECR ion source VENUS (18 and $28 \mathrm{GHz}$ ), which is currently 
under construction, will further enhance the capability of the 88-Inch cyclotron in terms of intensity and energy for the heavy ion beam program [5]. The ion optics design of a new high intensity injector beam line for VENUS has been developed over the last year [6]. To support the simulations and to identify possible upgrades of the existing beam lines, the ion optical parameters of the AECR-U injection line have been carefully evaluated. The injection beam line consists of an analyzing section (Glaser Lens and sector magnet), a horizontal matching section $\left(17.5^{\circ}\right.$ bend, Glaser lens, quadrupole doublet and $72.5^{\circ}$ bend) and the axial injection section (3 Glaser lenses). A layout of the horizontal and axial injection line is shown in Figure 2 and is described in detail in [7].

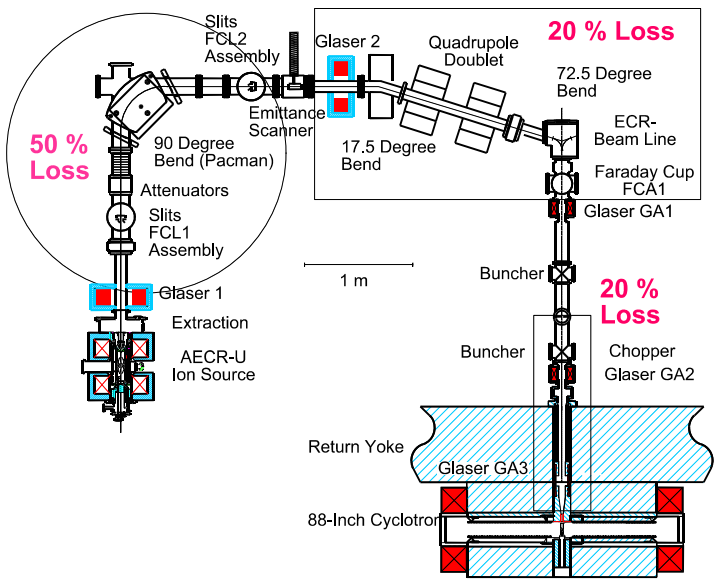

Figure 2: Layout of the horizontal and axial injection line. The horizontal section is rotated $90^{\circ}$ in the figure.

\subsection{Extraction and Ion Beam Formation}

The ion beam formation of the AECR has been simulated for an ion source condition typical for the production of a high intensity $\mathrm{Kr}^{19+}$ ion beam (analyzed current $60 \mathrm{e} \mu \mathrm{A}$ at $14 \mathrm{kV}$ extraction, total extracted ion beam current $1.5 \mathrm{~mA}$, including oxygen gas mixing). Only one charge state $\left(\mathrm{Kr}^{19+}\right)$ was used in the simulation. The contribution of all the other charge states and the oxygen mixing gas were taken into account by normalizing their current with respect to the desired $\mathrm{Kr}^{19+}$. This simulation method is described in detail in [8]. Figure 3 shows the dependence of the ion beam emittance on the extraction voltage simulated with identical plasma densities. The emittance reaches a minimum at extraction voltages above $25 \mathrm{kV}$. At extraction voltages above 40 $\mathrm{kV}$ the beam gets over-focused and the emittance is growing again. Unfortunately the insulation of the AECR-U only permits extraction voltages up to $15 \mathrm{kV}$ and upgrades are needed to extract at higher voltages.

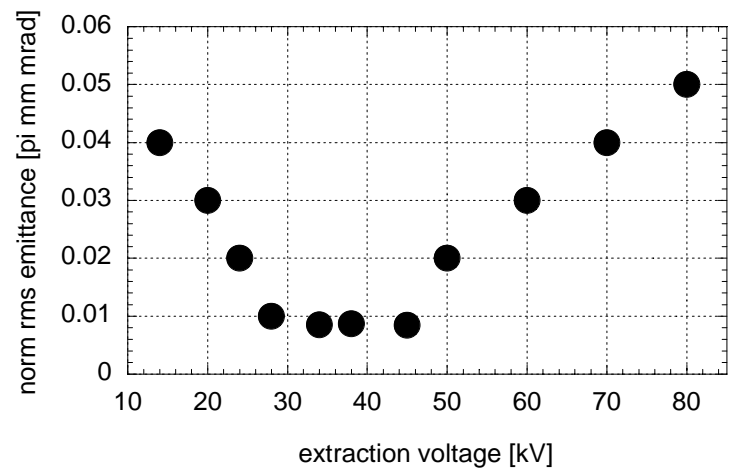

Figure 3: Dependence of the ion beam emittance on the extraction voltage simulated with identical plasma densities for the AECR-U ion source.

\subsection{Ion beam transport through the injection beamline}

Figure 2 indicates typical ion beam losses in the various sections of the AECR-U injection beam-line. The biggest losses are in the analyzing section. Up to $50 \%$ of the ion beam is lost in this section. Consequently by replacing the analyzing section (Glaser Lens and analyzing magnet) with a higher acceptance double focusing magnet would substantially increase the ion beam transmission.

With measured ion beam emittances (see section 2.4) as input, the ion beam optics of the horizontal and axial injection lines were investigated with TRACE3D [9] and TRANSOPTR [10], which include first order space charge effects. The 72.5-degree vertical bend magnet at the entrance to the axial injection line was identified as the weakest point in the horizontal and axial injection lines: The ion beam has to be focused strongly in the vertical plane to achieve a round and small divergence beam after that magnet. Therefore, the quadrupole doublet in front of the magnet is the most delicate tuning element in the beam line during operation. At higher intensities (e.g $50 \mathrm{e} \mu \mathrm{A} \mathrm{Kr}^{19+}$ ), beam matching to the axial beam line cannot be achieved due to the space charge forces counteracting the required strong focusing in the vertical plane, consequently leading to losses in both beam line sections. Simulations show that if the beam could be matched correctly to the axial beam line (requiring a redesign of the 72.5-degree bend magnet), currents of $120 \mathrm{e} \mu \mathrm{A} \mathrm{Kr}^{19+}$ could be transported without losses to the cyclotron inflector even at an injection voltage of $14 \mathrm{kV}$.

\subsection{Ion beam emittance measurements}

Systematic ion beam emittance measurements after the AECR-U analyzing magnet in both the horizontal ( $x^{\prime}$ ') and vertical (yy') plane were performed for a wide range of ions [11]. The results indicate a strong mass dependence of the normalized beam emittance. For example the normalized rms emittance for protons 
$(0.24 \pi \cdot \mathrm{mm} \cdot \mathrm{mrad})$ is four times higher than for $\mathrm{O}^{6+}(0.06$ $\pi \cdot \mathrm{mm} \cdot \mathrm{mrad})$ and about 8 times higher than $\mathrm{Kr}^{19+}(0.03$ $\pi \cdot \mathrm{mm} \cdot \mathrm{mrad})$. Figure 4 shows this mass dependence for ions from $\mathrm{H}$ to $\mathrm{Bi}$. Within a charge state distribution the emittance values decrease for higher charge states [11] even at equal current levels. For instance the ion beam emittance of $18.8 \mathrm{e} \mu \mathrm{A}$ of $\mathrm{Bi}^{21+}$ was measured to be $0.07 \pi \cdot \mathrm{mm} \cdot \mathrm{mrad}$, while the emittance of $18 \mathrm{e} \mu \mathrm{A}$ of $\mathrm{Bi}^{32+}$ was $0.03 \pi \cdot \mathrm{mm} \cdot \mathrm{mrad}$.

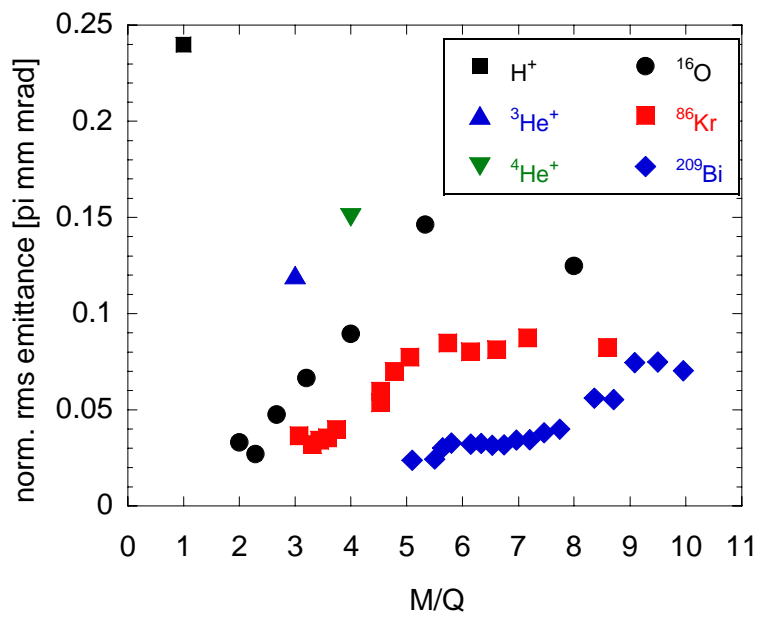

Figure 4: Comparison of emittance values for different masses and charge states.

The ion beam emittance is much more dependent on the actual plasma conditions than on the extracted ion beam intensities at low and medium ion beam currents. For instance typical $\mathrm{Ar}^{9+}$ normalized rms emittance values were measured to be $.067,0.064,0.065,0.058$ $\pi \cdot \mathrm{mm} \cdot \mathrm{mrad}$ at total extracted currents of $0.75,0.86,1.2$ and $2 \mathrm{emA}$, delivering 10, 52, 108 and $145 \mathrm{e} \mu \mathrm{A}$ of $\mathrm{Ar}^{9+}$ at $15 \mathrm{kV}$ extraction voltage. The emittance values changed less than $10 \%$ over the mentioned intensity range; the lowest emittance was actually measured at the highest current in this case. On the other hand, the ion beam emittance can easily change a factor of 2 or 3 at comparable ion beam intensities during unstable plasma conditions.

The condition of the plasma chamber wall strongly influences the plasma stability. If the source had been opened or the ion beam has been changed from one metal to another it can take 2 to 3 days until the lowest emittance values and peak performance can be achieved. Consequently during rapid beam changes involving high intensity metal ion beams, the tune has to be a compromise between higher beam intensity and lower beam emittance. In the year 2000 about $870 \mathrm{~h}$ of high intensity $\mathrm{Kr}^{19+}$ has been accelerated, and several times the beam emittance was measured. If the source was fully conditioned, the emittance value for $50 \mathrm{e} \mu \mathrm{A} \mathrm{Kr}^{19+}$ was $0.03 \pi \cdot \mathrm{mm} \cdot \mathrm{mrad}$. Following a beam change or after the source had been opened to air, emittance values as high as 0.07 to $0.09 \pi \cdot \mathrm{mm} \cdot \mathrm{mrad}$ have been measured for similar intensities. Therefore, the emittance measurement device can be used as an online ion source tuning aid.

\section{ION BEAM INJECTION INTO THE CYCLOTRON}

\subsection{Ion beam transmission}

Over the last three years, the cyclotron transmission (ratio of analyzed current to extracted cyclotron current) for high-intensity heavy ion beams has been significantly improved. New dee and dummy dee inserts have been designed to reduce the transit time in the first turn making the acceleration of $3^{\text {rd }}$ harmonic beams more efficient [12]. With an improved inflector design the injection energy could be raised from $10 \mathrm{kV}$ to $14 \mathrm{kV}$. The higher injection energy increased the beam line transmission throughout the injection line, but especially in the first analyzing section. At the same ion source condition, the analyzed ion beam current increased up to $30 \%$ for a single charge state. The extracted cyclotron beam improved up to $60 \%$ (see Figure 5). Figure 5 shows the result of a beam development run with argon using an injection voltage of $10 \mathrm{kV}$ and $15 \mathrm{kV}$. While nothing is gained at the very low intensities (analyzed beam of 10 e $\mu \mathrm{A} \mathrm{Ar}^{9+}$ ), substantial gains can be achieved for the medium intensity beams (40 to $60 \mathrm{e} \mu \mathrm{A})$. For the highest intensity beams even higher injection voltages would be needed.

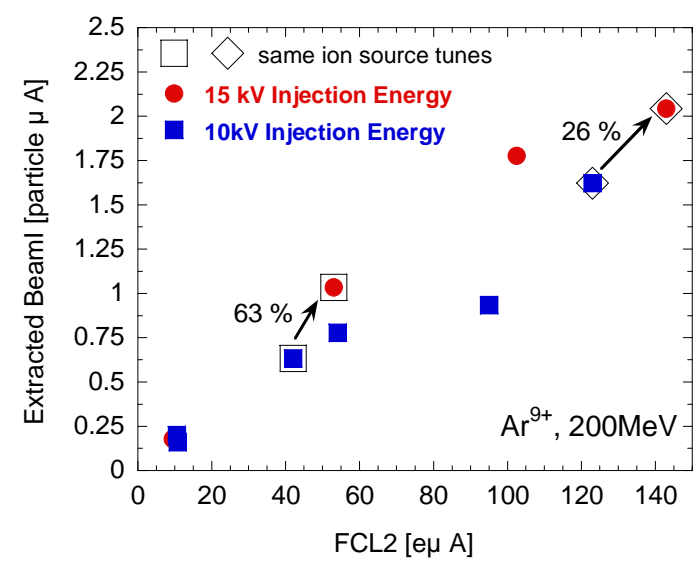

Figure 5: Dependence of the extracted cyclotron current from the analyzed current

Table 1 shows typical high intensity transmission through the injection line and the cyclotron for a $460 \mathrm{MeV} \mathrm{Kr}^{19+}$ beam with the new center region inserts.

\begin{tabular}{|l|l|}
\hline $450 \mathrm{MeV} \mathrm{Kr}$ & $19+$ \\
\hline Analyzing section & 50 to $60 \mathrm{e} \mu \mathrm{A}$ \\
\hline Horizontal matching section & .4 to .6 trans. \\
\hline Axial injection section & .7 to .85 trans. \\
\hline Beam capture and transport through 5” & .75 to .9 trans. \\
\hline Transmission from 5” to 35” 35 to .45 trans. \\
\hline Extraction efficiency & .8 to .95 trans. \\
\hline
\end{tabular}


Total extracted beam

7.5 to $9.4 \mathrm{e} \mu \mathrm{A}$

\subsection{Centering of the cyclotron Beam at higher injection voltages}

Target probe measurements have been performed to test the centering of the ion beam in the cyclotron for a higher injection voltage of $15 \mathrm{kV}$ (compared to the currently used $10 \mathrm{kV}$ ). Sufficient centering of the ion beam could be achieved for the higher injection voltage. Orbit tracking with Z3CYCLONE was performed for injection voltages up to $30 \mathrm{kV}$ and reasonable centering could be demonstrated. Hence the AECR-U insulation will be upgraded for extraction voltages up to $20 \mathrm{kV}$ and the extraction system of VENUS is designed for $30 \mathrm{kV}$.

\subsection{Sputtering of the Grid Wires}

The 88-Inch Cyclotron uses an electrostatic gridded mirror assembly to bend the ion beam into the median plane of the cyclotron. Failure of the electrostatic mirror grid is one of the main causes for interruption of long high intensity ion beam runs. The mirror grid is made of $0.06 \mathrm{~mm}$ diameter tungsten wire spaced $1 \mathrm{~mm}$ apart. The beam transmission through the grid is $90 \%$. The lifetime of the grid depends strongly on the mass and the intensity of the injected ion beam. Table 2 shows the sputter yield for different ions on tungsten. While the sputter yield for light ions is very low and the lifetime of an inflector assembly can be many days or weeks, sputtering becomes a serious issue for the heavier ions. For instance the inflector lifetime is typically reduced to 24 to 36 hours for a $50 \mathrm{e} \mathrm{A}$ beam of ${ }^{86} \mathrm{Kr}^{19+}$. Although a routine inflector change takes only about $30 \mathrm{~min}$, it is still an interruption of the experiment. Therefore, plans for the design of a new spiral inflector and the required redesign of the cyclotron center region are currently being evaluated.

Table 2: Sputter yields for different ions on tungsten at 10 to $30 \mathrm{keV}$ injection energy [13]

\begin{tabular}{|l|c|}
\hline \multicolumn{1}{|c|}{ Ion } & atoms/ion \\
\hline $\mathrm{H}^{+}$ & 0.002 \\
\hline $\mathrm{He}^{+}$ & 0.05 \\
\hline $\mathrm{O}^{5+}$ & 0.5 \\
\hline $\mathrm{Ne}^{6+}$ & 1 \\
\hline $\mathrm{Ar}^{9+}$ & 2 \\
\hline $\mathrm{Kr}^{19+}$ & 5 \\
\hline $\mathrm{Xe}^{28+}$ & 10 \\
\hline $\mathrm{Pb}^{43+}$ & 20 \\
\hline
\end{tabular}

\section{ACKNOWLEDGEMENT}

The authors would like to thank the 88-Inch Cyclotron operator group for their great support. This work has been supported by the U.S. Department of Energy under Contract No. DE-AC03-76SF00098.

\section{REFERENCES}

[1] V. Ninov, K.E. Gregorich, ENAM 98, edited by B.M. Sherril, D.J. Morissey, and C.N. Davids (AIP Woodbury, 1999), p. 7047041999

[2] Xie, Z.Q., Lyneis, C.M., Clark, D.J., Guy, A., Lundgren, S.A, Proceedings of the $15^{\text {th }}$ Intern. Conference on Cyclotrons and their Applications, Caen, France, 179, 1998

[3] D. J. Clark, Y. Jongen, C. M. Lyneis, Proc. of the $10^{\text {th }}$ Conference on Cyclotrons and their Applications, Michigan State University, MI, USA, 1984

[4] Z. Q. Xie and C. M. Lyneis, Proceedings of the $13^{\text {th }}$ International Workshop on ECR Ion Sources, Texas A\&M, College Station, USA, 16 (1997).

[5] M.A. Leitner, C.M. Lyneis, D. Wutte, C.E. Taylor and S.R. Abbott, Physica Scripta in print, and these conference proceedings

[6] M.A. Leitner, D. Wutte, C.M. Lyneis, paper submitted to the $9^{\text {th }}$ International Conferences on Ion Sources, to be published in RSI

[7] D. Wutte, M. A. Leitner, C. M. Lyneis, Proceedings of the Seventh European Particle Accelerator Conference, (EPAC 2000), 26-30 June 2000, Austria Center Vienna

[8] D. Wutte, M. Leitner, C. M. Lyneis, C. E. Taylor, Z. Q. Xie, Proceedings of the Heavy Ion Accelerator Technology Conference (HIAT'98), Argonne National Laboratory, Illinois, USA, 1998.

[9] TRACE3D Docum., K.R. Crandall and D. P. Rusthoi, LA-UR-97-886, LANL Report, 1997

[10]E.A. Heighway, and R.M. Hutchen, NIM 187, 89 (1981)

[11]D. Wutte, M.A. Leitner and C.M. Lyneis, Physica Scripta, in print

[12] Clark, D., Xie, Z. Q., internal note, LBNL, (1998)

[13] Matsunami, N. et al. Atomic Data and Nuclear Data Tables 31, 1 (1984) 